\title{
Conservation laws and symmetry transformations of the electromagnetic field with sources
}

\author{
Gerard Nienhuis* \\ Huygens-Kamerlingh Onnes Laboratory, Leiden University, P.O. Box 9504, 2300 RA Leiden, The Netherlands
}

(Received 22 January 2016; published 25 February 2016)

\begin{abstract}
In classical electrodynamics, the universal conservation laws of energy, momentum, and angular momentum are expressed by well-known continuity equations for the densities of these quantities. In the presence of charges and currents source terms must be added. These terms describe the exchange of energy and (linear or angular) momentum between field and matter. Recently, other conserved quantities of the electromagnetic field have been introduced and discussed. Examples are the pseudoscalars chirality and helicity, which are related to the handedness of the field. Even though these quantities have no obvious definition for matter, their conservation laws can still be presented in the form of continuity equations with source terms added. We show that these terms shed light on the interaction of chiral light with matter. A different role of conserved quantities is that they generate symmetry transformations of the system. The spatial transformations translation and rotation of the radiation field are generated by differential operators acting on mode functions. These operators are identical in form to the operators for the momentum and angular momentum of a quantum particle with spin 1. Also, for the total helicity and spin angular momentum of the field such operators on mode functions can be identified. A quite different picture arises in a quantum description of the electromagnetic field. The operator nature of the conserved quantities then arises from the commutation rules of photon creation and annihilation operators. We analyze the relation between these two pictures of symmetry transformations of the electromagnetic field.
\end{abstract}

DOI: 10.1103/PhysRevA.93.023840

\section{INTRODUCTION}

In classical physics, Noether's theorem is a powerful instrument for identifying the conserved quantities that arise from continuous symmetries of a Lagrangian system. Universal conserved quantities are the energy, momentum, and angular momentum. In quantum physics, the operators describing conserved quantities are represented by linear operators that commute with the Hamiltonian. These operators generate the corresponding symmetry transformation of the quantum states or of the quantum operators. For instance, the operators for the energy, momentum, and angular momentum not only represent observable quantities, but also occur in the description of the time evolution, spatial translation, and rotation of quantum states. These are the universal space-time symmetry transformations.

In the special case of electromagnetic fields there are conserved quantities other than the energy, momentum, and angular momentum. One example is the optical chirality. This is a pseudoscalar, which has been introduced as a measure of the handedness of the field [1-5]. It is a member of a class of conserved quantities introduced by Lipkin [6]. A closely related quantity is the optical helicity [7-10], which has recently received attention also in relation to the (im)possibility of properly identifying a spin part of the total angular momentum [11-16]. Most of these works focus on classical fields and discuss the expressions of the densities of the conserved quantities in coordinate space or in Fourier space.

For the free Maxwell fields all mentioned conserved quantities can be properly expressed as the volume integral of a density. These densities, which depend on the position and time, are quadratic functions of the local electric and magnetic

\footnotetext{
"nienhuis@physics.leidenuniv.nl
}

fields $\mathbf{E}$ and $\mathbf{B}$. They obey a continuity equation, so that the integrated quantity is a constant of the motion. For radiation fields one is usually only interested in the average of these densities over an oscillation time, but in fact, when properly defined, they should obey exact continuity equations. When also sources (charges and currents) are present, the energy and momentum can be exchanged between fields and matter, so that the continuity equations contain loss or gain terms, which correspond to the exchange of energy and momentum between radiation and matter. In contrast, the pseudoscalars chirality and helicity are not defined for matter. Nevertheless, we can derive expressions for the rates of loss or gain of the field quantities. These expressions determine the role of charges and currents as sources or sinks of helicity and chirality.

We also consider the role of these conserved quantities as generators of symmetry transformations. The classical radiation field is described by transverse (divergence-free) vector functions, representing the magnetic field and the transverse part of the electric field. Symmetry transformations of classical fields can be handled in the same way as transformations of wave functions of a quantum particle. Therefore, it is attractive to view the classical radiation field as the wave function of a photon in coordinate representation [17]. After quantization the expressions for the conserved quantities contain products of creation and annihilation operators, multiplied by matrix elements of the differential operators describing the classical transformations. We discuss the analogy and difference between these two representations of symmetry transformations.

\section{CONTINUITY OF ENERGY AND MOMENTUM}

For comparison with other conservation laws, and to fix the notation, we first summarize the well-known continuity equations for the energy and momentum. We take the microscopic view, where charges and currents are carried by point particles, so that the charge density $\rho$ and the current density $\mathbf{j}$ are linear 
combinations of the delta functions $\delta_{3}\left(\mathbf{r}-\mathbf{r}_{m}\right)$ for a particle with position $\mathbf{r}_{m}$. Then we only have to consider the fields in vacuum, so that Maxwell's equations have the simple form [18]

$$
\begin{gathered}
\nabla \cdot \mathbf{E}=\frac{\rho}{\epsilon_{0}}, \quad \nabla \times \mathbf{E}+\frac{\partial \mathbf{B}}{\partial t}=0, \\
\nabla \cdot \mathbf{B}=0, \quad c^{2} \nabla \times \mathbf{B}=\frac{\partial \mathbf{E}}{\partial t}+\frac{\mathbf{j}}{\epsilon_{0}} .
\end{gathered}
$$

Symmetry transformations of fields can be handled in the same way as transformations of wave functions of a quantum particle. For instance, translations are generated by the derivative with respect to position, which determines also the momentum operator on the wave function.

According to the Maxwell theory of classical electrodynamics, the expressions for the energy density $u$ and the momentum density $\mathbf{p}$ of the radiation field are given by the relations

$$
u=\frac{\epsilon_{0}}{2}\left(\mathbf{E}^{2}+c^{2} \mathbf{B}^{2}\right), \quad \mathbf{p}=\epsilon_{0} \mathbf{E} \times \mathbf{B} .
$$

The energy density is the sum of an electric and a magnetic contribution, while a nonzero momentum density requires the presence of both an electric and a magnetic field. We also recall the expression for the Maxwell stress tensor T [18]:

$$
\mathrm{T} \equiv \epsilon_{0}\left(\mathbf{E E}+c^{2} \mathbf{B B}\right)-u \mathrm{l} .
$$

The symmetric second-rank stress tensor $\mathrm{T}$ with components $\mathrm{T}_{i j}, i, j=x, y, z$, may be viewed as the opposite of the momentum flux density, so that for a unit vector $\hat{\mathbf{n}}$ the vector $-\hat{\mathbf{n}} \cdot \mathrm{T}$ is the field momentum flowing through a unit area normal to $\hat{\mathbf{n}}$. The unit tensor I has components $\mathrm{I}_{i j}=\delta_{i j}$.

The well-known continuity equations for the densities, (2), follow directly by taking the time derivatives of these quantities and eliminating the time derivatives of the field by using Maxwell's equations. The significance of $c^{2} \mathbf{p}$ as the energy flux density (the Poynting vector) follows from the resulting continuity equation for the energy density:

$$
\frac{\partial u}{\partial t}+c^{2} \nabla \cdot \mathbf{p}=-w .
$$

Here

$$
w=\mathbf{j} \cdot \mathbf{E}
$$

is the density of the work that is exerted by the fields on the moving charges or, equivalently, the power density lost by the fields. Hence, Eq. (4) expresses the energy conservation of fields and sources combined. We emphasize that no exact continuity equations hold separately for the electric and the magnetic contribution to the energy density.

The continuity equation for the momentum is found as

$$
\frac{\partial \mathbf{p}}{\partial t}-\nabla \cdot \mathbf{T}=-\mathbf{f},
$$

where

$$
\mathbf{f}=\rho \mathbf{E}+\mathbf{j} \times \mathbf{B}
$$

is the force density on the sources, while $-\mathbf{f}$ is the loss rate of the field momentum density. The result, (6), expresses the conservation of the total momentum, with $-\mathrm{T}$ the momentum flux density of the fields. For stationary fields (with $\partial \mathbf{p} / \partial t=0$ ), the total force on charges and currents inside a volume equals the integral of T over the surface of the volume, which explains its nature as the stress tensor.

These continuity equations, (4) and (6), are exact consequences of Maxwell's equations, which hold instantaneously for each position. No averages over a wavelength or an oscillation time are needed. For instance, for a monochromatic field in a source-free region, we denote the fields $\mathbf{E}(\mathbf{r}, t)=\operatorname{Re} \tilde{\mathbf{E}}(\mathbf{r}) \exp (-i \omega t)$ and $\mathbf{B}(\mathbf{r}, t)=\operatorname{Re} \tilde{\mathbf{B}}(\mathbf{r}) \exp (-i \omega t)$, for complex fields $\tilde{\mathbf{E}}(\mathbf{r})$ and $\tilde{\mathbf{B}}(\mathbf{r})$. For a monochromatic field the expressions for the density of energy and momentum take the form

$$
\begin{aligned}
& u_{\text {mon }}=\frac{\epsilon_{0}}{4} \operatorname{Re}\left[\tilde{\mathbf{E}}^{*} \cdot \tilde{\mathbf{E}}+c^{2} \tilde{\mathbf{B}}^{*} \cdot \tilde{\mathbf{B}}+\left(\tilde{\mathbf{E}}^{2}+c^{2} \tilde{\mathbf{B}}^{2}\right) e^{-2 i \omega t}\right] \\
& \mathbf{p}_{\text {mon }}=\frac{\epsilon_{0}}{2} \operatorname{Re}\left[\tilde{\mathbf{E}} \times \tilde{\mathbf{B}}^{*}+\tilde{\mathbf{E}} \times \tilde{\mathbf{B}} e^{-2 i \omega t}\right] .
\end{aligned}
$$

The last terms in these expressions have a phase that is rapidly oscillating in space and time. This limits the common analogy between the fields in classical electrodynamics and the wave function of a quantum particle [17]. In this picture, a monochromatic field is the natural analog of a stationary quantum state. However, while all expectation values of a quantum observable in a stationary state are independent of time, the densities of energy and momentum of a monochromatic field contain rapid oscillations at the double frequency $2 \omega$. Only in special cases, such as circularly polarized plane waves, are $u_{\text {mon }}$ and $\mathbf{p}_{\text {mon }}$ uniform in space and constant in time.

\section{CHIRALITY AND HELICITY}

\section{A. Continuity of the chirality and its flux}

The optical chirality has been introduced as a measure of the handedness of an electromagnetic field. The density of the chirality is defined in terms of the local electromagnetic field by [1-3]

$$
\chi=\frac{\epsilon_{0}}{2}\left[\mathbf{E} \cdot(\nabla \times \mathbf{E})+c^{2} \mathbf{B} \cdot(\nabla \times \mathbf{B})\right] .
$$

This quantity changes sign under parity transformation, so that it is a pseudoscalar. It consists of a magnetic and an electric part. A large chirality can confer enhanced selectivity between two enantiomers in excitation or absorption by chiral molecules [19-21]. This might be used for strategies to separate chiral systems into their enantiomers. Lipkin noted earlier that the chirality is a conserved quantity of the free field, along with a hierarchy of other quantities [6]. The appropriate expression for the chirality flux density is given by the pseudovector $[2,3]$

$$
\boldsymbol{\phi}=\frac{\epsilon_{0} c^{2}}{2}[\mathbf{E} \times(\nabla \times \mathbf{B})-\mathbf{B} \times(\nabla \times \mathbf{E})] .
$$

In order to derive the continuity equation for the chirality density $\chi$, we take its time derivative, while using Maxwell's equations, (1). This produces the exact equality [1]

$$
\frac{\partial \chi}{\partial t}+\nabla \cdot \boldsymbol{\phi}=-\frac{1}{2}[\mathbf{j} \cdot(\nabla \times \mathbf{E})+\mathbf{E} \cdot(\nabla \times \mathbf{j})] .
$$

Such a continuity equation does not hold separately for the electric and the magnetic part of the chirality, (9). For an electromagnetic field in a source-free region, the right-hand 
side of (11) vanishes, so that the chirality is a locally conserved quantity.

It is tempting to compare the continuity equations, (4) for energy and (11) for chirality. Both densities, $u$ and $\chi$, are the sum of an electric and a magnetic term, which are defined in terms of the local fields. Both continuity equations hold exactly for each position and time, without any averaging. In both cases, the electric and magnetic terms separately do not obey a continuity equation. The loss term in both cases contains a coupling between the electric field and the current density. In Eq. (4) this scalar term is directly identified as the field energy lost to the sources. The right-hand side of (11) indicates that the quantity $[\mathbf{j} \cdot(\nabla \times \mathbf{E})+\mathbf{E} \cdot(\nabla \times \mathbf{j})] / 2$ may be viewed as the loss rate of the chirality density, due to a pseudoscalar coupling of the electric field and the current density. This coupling is proportional to the current in the direction of the curl of the electric field, or vice versa. The total chirality loss rate is found by integrating the density loss rate over space. Integration by parts shows that the two terms give the same contribution, and the total chirality loss rate is equal to

$$
\int d_{3} \mathbf{r} \mathbf{j} \cdot(\nabla \times \mathbf{E})=\int d_{3} \mathbf{r} \mathbf{E} \cdot(\nabla \times \mathbf{j})
$$

In contrast to the energy case, this loss (or gain) of chirality of the field is not compensated by a corresponding gain (or loss) by the sources.

Another striking difference between the density of the energy and that of the chirality arises in the important special case of monochromatic fields in a source-free region. Then the complex fields $\tilde{\mathbf{E}}$ and $\tilde{\mathbf{B}}$ are related by $\nabla \times \tilde{\mathbf{E}}=i \omega \tilde{\mathbf{B}}$ and $\nabla \times \tilde{\mathbf{B}}=-i \omega \tilde{\mathbf{E}} / c^{2}$. For the densities of the chirality and chirality flux this gives the results

$$
\chi_{\text {mon }}=\frac{\epsilon_{0} \omega}{2} \operatorname{Im} \tilde{\mathbf{E}} \cdot \tilde{\mathbf{B}}^{*}, \quad \boldsymbol{\phi}_{\mathrm{mon}}=\frac{\epsilon_{0} \omega}{4 i}\left[\tilde{\mathbf{E}}^{*} \times \tilde{\mathbf{E}}+c^{2} \tilde{\mathbf{B}}^{*} \times \tilde{\mathbf{B}}\right] .
$$

Hence, for monochromatic fields the density of the chirality and chirality flux are constant in time. This implies that, for a radiation field with a narrow frequency width $\Delta \omega$, the quantities $\chi$ and $\phi$ vary only on time scales of the order of $1 / \Delta \omega$.

In order to compare the continuity of the momentum density and the chirality flux density, we take the time derivative of $\boldsymbol{\phi}$. By using Maxwell's equations one obtains the exact continuity equation,

$$
\begin{aligned}
\frac{\partial \phi}{\partial t}-\nabla \cdot U= & -\frac{c^{2}}{2}[\mathbf{j} \times(\nabla \times \mathbf{B})-\mathbf{B} \times(\nabla \times \mathbf{j}) \\
& +\rho(\nabla \times \mathbf{E})],
\end{aligned}
$$

with $\bigcup$ the symmetric tensor,

$$
\begin{aligned}
\mathrm{U} \equiv & \frac{\epsilon_{0} c^{2}}{2}[(\nabla \times \mathbf{E}) \mathbf{E}+\mathbf{E}(\nabla \times \mathbf{E}) \\
& \left.+c^{2}(\nabla \times \mathbf{B}) \mathbf{B}+c^{2} \mathbf{B}(\nabla \times \mathbf{B})\right]-c^{2} \chi \mathbf{I} .
\end{aligned}
$$

This tensor resembles the stress tensor, (3), where now half of the field factors $\mathbf{E}$ and $\mathbf{B}$ are replaced by their curl. The loss term on the right-hand side of Eq. (14) contains a pseudovector coupling of the current density with the (curl of the) magnetic field and a coupling of the charge density with the curl of the electric field.

\section{B. Continuity of the optical helicity and its flux}

The concept of helicity in physics has various meanings. In a monochromatic optical field with a complex normalized polarization vector $\mathbf{e}$, the term helicity is used for the size of the vector $\left(\mathbf{e}^{*} \times \mathbf{e}\right) / i$. It is a number between -1 and 1 and represents the local degree of circular polarization. In quantum field theory, the helicity of a massless particle is the component of spin in the propagation direction. More generally, for any vector field $\mathbf{v}$, the pseudoscalar quantity $\mathbf{v} \cdot(\nabla \times \mathbf{v})$ quantifies the local twist of the field lines. When $\mathbf{v}$ is the velocity field of a fluid, the integral $\int d_{3} \mathbf{r} \mathbf{v} \cdot(\nabla \times \mathbf{v})$ has been called the vortex helicity [22]. The concepts of optical chirality and helicity have a character similar to this vortex helicity.

All densities discussed so far have a local character, in the sense that they are fully defined in terms of the local electric and magnetic fields and their curl. This is no longer true for the density of the optical helicity. It can be defined in analogy with the chirality, (9), with the electric and magnetic fields replaced by corresponding vector potentials. In order to introduce an electric vector potential, we first separate the electric field into its transverse and its longitudinal part. We denote

$$
\mathbf{E}=\mathbf{E}_{\perp}+\mathbf{E}_{\|},
$$

where the transverse part $\mathbf{E}_{\perp}$ has a vanishing divergence, and the longitudinal part $\mathbf{E}_{\|}$a vanishing curl. The field $\mathbf{E}_{\|}$is then identical to the Coulomb field corresponding to the instantaneous charge distribution [23], while the transverse part $\mathbf{E}_{\perp}$ may be viewed as the radiative electric field. This separation in the Coulomb field and the radiation field is inevitable in a quantized description of the electromagnetic field with sources. The potentials are uniquely defined by the relations

$$
\mathbf{B}=\nabla \times \mathbf{A}, \mathbf{E}_{\perp}=-\nabla \times \mathbf{C},
$$

provided that we impose the additional requirement that they are purely transverse, so that $\boldsymbol{\nabla} \cdot \mathbf{A}=0$ and $\boldsymbol{\nabla} \cdot \mathbf{C}=0$. In Fourier space, the separation of the electric field in a transverse and a longitudinal part is local and corresponds to the separation of the vector field in components normal and parallel to the vector $\mathbf{k}$. This implies that the separation is nonlocal in ordinary space. By the same token, the vector potentials $\mathbf{C}$ and $\mathbf{A}$ are not locally determined by the fields, but their value at one position depends on $\mathbf{E}$ or $\mathbf{B}$ at other positions.

We indicate quantities determined by the transverse fields $\mathbf{E}_{\perp}$ and $\mathbf{B}$ by the suffix "rad." The helicity density of the radiation field is defined by $[24,25]$

$$
\begin{aligned}
h_{\mathrm{rad}} & =\frac{\epsilon_{0}}{2 c}\left[\mathbf{C} \cdot(\nabla \times \mathbf{C})+c^{2} \mathbf{A} \cdot(\nabla \times \mathbf{A})\right] \\
& =\frac{\epsilon_{0}}{2 c}\left(c^{2} \mathbf{A} \cdot \mathbf{B}-\mathbf{C} \cdot \mathbf{E}_{\perp}\right) .
\end{aligned}
$$

This generalizes an earlier definition of a purely magnetic helicity density (proportional to $\mathbf{A} \cdot \mathbf{B}$ ) by Woltjer [26]. In plasma physics, this magnetic term is the relevant one [27]. However, in general the magnetic term alone does not obey an exact continuity equation, whereas expression (18) does. A simple way to derive this is by noting that Maxwell's equations 
in terms of the potentials take the form

$$
-\nabla \times \mathbf{C}+\frac{\partial \mathbf{A}}{\partial t}=0, \quad c^{2} \nabla \times \mathbf{A}=-\frac{\partial \mathbf{C}}{\partial t}+\frac{\mathbf{g}}{\epsilon_{0}},
$$

where we have defined the transverse current density $\mathbf{g}$ by the requirement that $\mathbf{j}_{\perp}=\nabla \times \mathbf{g}$. These equations of motion have the same form as the transverse part of Maxwell's equations with the substitutions $\mathbf{B} \rightarrow \mathbf{A}, \mathbf{E}_{\perp} \rightarrow-\mathbf{C}$, and $\mathbf{j}_{\perp} \rightarrow \mathbf{g}$. These same substitutions applied to the expression, (9), for the chirality density produce the definition, (18), of $h_{\text {rad }}$. It follows that $h_{\text {rad }}$ obeys the exact continuity equation,

$$
\frac{\partial h_{\mathrm{rad}}}{\partial t}+\nabla \cdot \mathbf{v}_{\mathrm{rad}}=\frac{1}{2 c}[\mathbf{g} \cdot(\boldsymbol{\nabla} \times \mathbf{C})+\mathbf{C} \cdot(\boldsymbol{\nabla} \times \mathbf{g})],
$$

with the helicity flux density

$$
\begin{aligned}
\mathbf{v}_{\text {rad }} & =\frac{\epsilon_{0} c}{2}[\mathbf{A} \times(\nabla \times \mathbf{C})-\mathbf{C} \times(\nabla \times \mathbf{A})] \\
& =\frac{\epsilon_{0} c}{2}\left[\mathbf{E}_{\perp} \times \mathbf{A}+\mathbf{B} \times \mathbf{C}\right] .
\end{aligned}
$$

The right-hand side of Eq. (20) specifies the loss of helicity. This loss arises exclusively from the transverse part of the current density. For a free field, the helicity is a locally conserved quantity [28]. In analogy with the expression, (12), for the chirality loss, the integrated helicity loss rate can be written as

$$
-\frac{1}{c} \int d_{3} \mathbf{r} \mathbf{g} \cdot(\nabla \times \mathbf{C})=-\frac{1}{c} \int d_{3} \mathbf{r} \mathbf{C} \cdot(\nabla \times \mathbf{g}) .
$$

A continuity equation for the helicity flux density is obtained by taking the time derivative of $\mathbf{v}_{\text {rad }}$, while using Maxwell's equations. This gives

$$
\frac{\partial \mathbf{v}_{\mathrm{rad}}}{\partial t}-\nabla \cdot \mathbf{W}=-\frac{c}{2}[\mathbf{g} \times(\nabla \times \mathbf{A})-\mathbf{A} \times(\nabla \times \mathbf{g})],
$$

with $\mathrm{W}$ the symmetric tensor

$$
\mathbf{W} \equiv \frac{\epsilon_{0} c}{2}\left[c^{2}(\mathbf{A B}+\mathbf{B A})-\mathbf{C E}_{\perp}-\mathbf{E}_{\perp} \mathbf{C}\right]-c^{2} h_{\mathrm{rad}} \mathrm{l} .
$$

Again, this equation holds exactly, without any averaging over time or space.

The relation between chirality and helicity greatly simplifies in the important special case of free monochromatic fields. In that case, it follows from (18) and (21) that $h_{\mathrm{rad}}=c \chi / \omega^{2}$ and $\mathbf{v}_{\text {rad }}=c \boldsymbol{\phi} / \omega^{2}$. All these quantities are constant in time. Moreover, the expressions for the flux densities $\mathbf{v}_{\text {rad }}$ and $\boldsymbol{\phi}_{\mathrm{rad}}$ in the monochromatic case are proportional to the local degree of circular polarization, which relates them to the optical spin.

One should note that the helicity density $h_{\text {rad }}$ and the helicity flux density $\mathbf{v}_{\text {rad }}$ are defined in terms of the vector potentials, which are not generally determined by the local electric and magnetic fields. This is not particularly worrying. In fact, also for a massless quantum particle the concept of helicity as the spin in the propagation direction has an inherent nonlocal character.

We have shown that exact continuity equations hold for the energy, chirality, and helicity and their fluxes, without averaging over space or time. A requirement for this to be true is that these quantities are defined to respect the dual electric-magnetic symmetry $[13,28]$, which implies that they are invariant under the substitutions for the fields

$$
\begin{aligned}
\mathbf{E}_{\perp} \rightarrow \mathbf{E}_{\perp}^{\prime} & =\mathbf{E}_{\perp} \cos \phi+c \mathbf{B} \sin \phi, \\
c \mathbf{B} \rightarrow c \mathbf{B}^{\prime} & =-\mathbf{E}_{\perp} \sin \phi+c \mathbf{B} \cos \phi .
\end{aligned}
$$

The corresponding substitutions for the potentials are

$$
\begin{aligned}
\mathbf{C} \rightarrow \mathbf{C}^{\prime} & =\mathbf{C} \cos \phi-c \mathbf{A} \sin \phi, \\
c \mathbf{A} \rightarrow c \mathbf{A}^{\prime} & =\mathbf{C} \sin \phi+c \mathbf{A} \cos \phi .
\end{aligned}
$$

\section{QUANTUM AND CLASSICAL OPERATORS FOR CONSERVED QUANTITIES}

\section{A. Quantized radiation field}

A standard route of quantization of the radiation field starts by expanding the fields in orthogonal modes $\mathbf{G}_{\lambda}(\mathbf{r})$ [23]. For convenience we assume that the mode index is discrete. Generalization to a continuum of modes is straightforward. The modes form a complete orthonormal set of complex divergence-free vector solutions of Helmholtz's equation $\nabla^{2} \mathbf{G}_{\lambda}+k_{\lambda}^{2} \mathbf{G}_{\lambda}^{2}=0$, where $k_{\lambda}$ defines the mode frequency $\omega_{\lambda}=c k_{\lambda}$. Because of the transversality of the modes, they obey the identity $\nabla \times\left(\nabla \times \mathbf{G}_{\lambda}\right)=k_{\lambda}^{2} \mathbf{G}_{\lambda}$. The modes span a Hilbert space $\mathbb{M}$ of modes with the scalar product $\left\langle\mathbf{G} \mid \mathbf{G}^{\prime}\right\rangle=$ $\int d_{3} \mathbf{r} \mathbf{G}^{*}(\mathbf{r}) \cdot \mathbf{G}^{\prime}(\mathbf{r})$. The orthonormality condition of modes is specified by $\left\langle\mathbf{G}_{\lambda} \mid \mathbf{G}_{\lambda^{\prime}}\right\rangle=\delta_{\lambda \lambda^{\prime}}$. Notwithstanding the Dirac notation, the concepts of modes and mode space have a completely classical significance.

When the vector potential $\mathbf{A}$ and the radiative (transverse) electric field $\mathbf{E}_{\perp}$ are expanded in the modes, the expansion coefficients are mathematically equivalent to harmonic oscillators, driven by the sources. Quantization of the fields is then equivalent to quantization of these harmonic oscillators, which turns the mode amplitudes into ladder operators and the fields into quantum field operators. Operators are indicated by a caret over the symbol. The resulting Hermitian operators $\hat{\mathbf{A}}=\hat{\mathbf{A}}^{+}+$H.c. and $\hat{\mathbf{E}}_{\perp}=\hat{\mathbf{E}}_{\perp}^{+}+$H.c. are determined by their absorptive parts [23],

$$
\begin{aligned}
& \hat{\mathbf{A}}^{+}=\sum_{\lambda} \sqrt{\frac{\hbar}{2 \epsilon_{0} c k_{\lambda}}} \hat{a}_{\lambda} \mathbf{G}_{\lambda}, \\
& \hat{\mathbf{E}}_{\perp}^{+}=i \sum_{\lambda} \sqrt{\frac{\hbar c k_{\lambda}}{2 \epsilon_{0}}} \hat{a}_{\lambda} \mathbf{G}_{\lambda},
\end{aligned}
$$

with $\hat{a}_{\lambda}$ the annihilation operator for a photon in the mode $\mathbf{G}_{\lambda}$. The Hermitian conjugates are the corresponding emissive parts of the fields $\hat{\mathbf{A}}^{-}=\left(\hat{\mathbf{A}}^{+}\right)^{\dagger}, \hat{\mathbf{E}}_{\perp}^{-}=\left(\hat{\mathbf{E}}_{\perp}^{+}\right)^{\dagger}$. The longitudinal (curlfree) part $\mathbf{E}_{\|}$of the electric field is instantaneously linked to the charge distribution, and therefore it belongs to the degrees of freedom of the particles. We do not discuss the quantum description of the particles here.

We also need the expressions for the Hermitian operators $\hat{\mathbf{B}}=\nabla \times \hat{\mathbf{A}}$ for the magnetic field and $\hat{\mathbf{C}}$ for the electric vector potential, such that $\hat{\mathbf{E}}_{\perp}=-\nabla \times \hat{\mathbf{C}}$. These operators are likewise determined by their absorptive parts

$$
\hat{\mathbf{B}}^{+}=\sum_{\lambda} \sqrt{\frac{\hbar}{2 \epsilon_{0} c k_{\lambda}}} \hat{a}_{\lambda} \nabla \times \mathbf{G}_{\lambda},
$$




$$
\hat{\mathbf{C}}^{+}=-i \sum_{\lambda} \sqrt{\frac{\hbar c}{2 \epsilon_{0} k_{\lambda}^{3}}} \hat{a}_{\lambda} \nabla \times \mathbf{G}_{\lambda} .
$$

The quantum nature of the fields arises exclusively from the annihilation operators $\hat{a}_{\lambda}$ and their Hermitian conjugates $\hat{a}_{\lambda}^{\dagger}$, which are the photon creation operators. These operators obey the bosonic commutation rules $\left[\hat{a}_{\lambda^{\prime}}, \hat{a}_{\lambda}^{\dagger}\right]=\delta_{\lambda^{\prime} \lambda}$. They act on the Hilbert space $\mathbb{S}$ of states of the quantized field, which has as its basis the tensor products of number states $\left|n_{\lambda}\right\rangle$. These are eigenstates of the number operators $\hat{N}_{\lambda} \equiv \hat{a}_{\lambda}^{\dagger} \hat{a}_{\lambda}$, with eigenvalue $n_{\lambda}$.

\section{B. Quantum operators for conserved quantities}

In order to discuss the role of conserved quantities in generating symmetry transformations of the radiation field, we need the corresponding quantum operators. We start from the classical expressions for the densities and replace the fields with their quantum counterparts, which are defined by (27)-(30). We restrict ourselves to the radiation field, and therefore it is sufficient to use the quantum operator $\hat{\mathbf{E}}_{\perp}$ for the transverse electric field. The quantum operators for the densities of energy and momentum of the radiation field are

$$
\hat{u}_{\mathrm{rad}}=\frac{\epsilon_{0}}{2}\left(\hat{\mathbf{E}}_{\perp}^{2}+c^{2} \hat{\mathbf{B}}^{2}\right), \quad \hat{\mathbf{p}}_{\mathrm{rad}}=\epsilon_{0} \hat{\mathbf{E}}_{\perp} \times \hat{\mathbf{B}} .
$$

The operators for the total conserved quantities arise after integration over position. The quantum operator representing the total radiation energy $\hat{U}_{\text {rad }}=\int d_{3} \mathbf{r} \hat{u}_{\text {rad }}$ is then found in the intuitive form

$$
\hat{U}_{\mathrm{rad}}=\frac{1}{2} \sum_{\lambda} \hbar c k_{\lambda}\left[\hat{a}_{\lambda} \hat{a}_{\lambda}^{\dagger}+\hat{a}_{\lambda}^{\dagger} \hat{a}_{\lambda}\right]=\sum_{\lambda} \hbar c k_{\lambda}\left[\hat{N}_{\lambda}+\frac{1}{2}\right],
$$

where we have used the orthonormality of the modes $\mathbf{G}_{\lambda}$ and applied integration by parts. The electric and the magnetic contributions to the energy operator are quite similar. However, both contributions also contain terms proportional to two annihilation or two creation operators, $\hat{a}_{\lambda^{\prime}} \hat{a}_{\lambda}$ or $\hat{a}_{\lambda^{\prime}}^{\dagger} \hat{a}_{\lambda}^{\dagger}$. These terms only cancel when the electric and magnetic contributions are added.

In the same way we obtain the quantum vector operator for the total momentum of the radiation field $\hat{\mathbf{P}}_{\text {rad }}=\int d_{3} \mathbf{r} \hat{\mathbf{p}}_{\text {rad }}$. This gives

$$
\hat{\mathbf{P}}_{\mathrm{rad}}=\frac{\hbar}{2 i} \sum_{\lambda \lambda^{\prime}}^{\prime}\left[\hat{a}_{\lambda^{\prime}} \hat{a}_{\lambda}^{\dagger}+\hat{a}_{\lambda}^{\dagger} \hat{a}_{\lambda^{\prime}}\right] \int d_{3} \mathbf{r} \mathbf{G}_{\lambda}^{*} \cdot(\nabla) \mathbf{G}_{\lambda^{\prime}} .
$$

Each component of $\hat{\mathbf{P}}_{\text {rad }}$ corresponds to a component of the gradient operator $\nabla$. The dot on the right-hand side of Eq. (33) indicates the scalar product between the vector fields $\mathbf{G}_{\lambda}^{*}$ and $\mathbf{G}_{\lambda^{\prime}}$. Since modes with different wave numbers $k_{\lambda}$ are orthogonal, only pairs of indices $\lambda$ and $\lambda^{\prime}$ for which $k_{\lambda}=k_{\lambda^{\prime}}$ contribute. This is indicated by the prime on the summation $\sum^{\prime}$.

The vector operator for the total angular momentum of the radiation field is found from the integral $\mathbf{J}_{\text {rad }}=\int d_{3} \mathbf{r}(\mathbf{r} \times$ $\mathbf{p}_{\text {rad }}$ ), after substituting the quantum expressions for the fields $\mathbf{E}_{\perp}$ and $\mathbf{B}$ and applying integration by parts. This gives the

$$
\begin{aligned}
& \text { result } \hat{\mathbf{J}}_{\mathrm{rad}}=\hat{\mathbf{L}}_{\mathrm{rad}}+\hat{\mathbf{S}}_{\mathrm{rad}} \text {, with [11] } \\
& \qquad \hat{\mathbf{L}}_{\mathrm{rad}}=\frac{\hbar}{2 i} \sum_{\lambda \lambda^{\prime}}^{\prime}\left[\hat{a}_{\lambda^{\prime}} \hat{a}_{\lambda}^{\dagger}+\hat{a}_{\lambda}^{\dagger} \hat{a}_{\lambda^{\prime}}\right] \int d_{3} \mathbf{r} \mathbf{G}_{\lambda}^{*} \cdot(\mathbf{r} \times \nabla) \mathbf{G}_{\lambda^{\prime}}
\end{aligned}
$$

and

$$
\hat{\mathbf{S}}_{\mathrm{rad}}=\sum_{\lambda \lambda^{\prime}}^{\prime} \frac{\hbar}{2 i}\left[\hat{a}_{\lambda^{\prime}} \hat{a}_{\lambda}^{\dagger}+\hat{a}_{\lambda}^{\dagger} \hat{a}_{\lambda^{\prime}}\right] \int d_{3} \mathbf{r}\left[\mathbf{G}_{\lambda}^{*} \times \mathbf{G}_{\lambda^{\prime}}\right] .
$$

It is quite natural to consider this result for the radiative angular momentum as a separation into an orbital part $\hat{\mathbf{L}}_{\text {rad }}$ and a spin part $\hat{\mathbf{S}}_{\text {rad }}$.

Starting from Eq. (18), the quantum operator for the total helicity $\hat{H}_{\text {rad }}$ is obtained in the same way. After substituting expressions (27)-(30) for the field operators and performing the integration over $\mathbf{r}$, we arrive at the result

$$
\hat{H}_{\mathrm{rad}}=\sum_{\lambda \lambda^{\prime}}^{\prime} \frac{\hbar}{2 k_{\lambda}}\left[\hat{a}_{\lambda^{\prime}} \hat{a}_{\lambda}^{\dagger}+\hat{a}_{\lambda}^{\dagger} \hat{a}_{\lambda^{\prime}}\right] \int d_{3} \mathbf{r} \mathbf{G}_{\lambda}^{*} \cdot\left[\nabla \times \mathbf{G}_{\lambda^{\prime}}\right]
$$

The operator for the integrated helicity flux density can be found in the same way, starting from (21). Remarkably, the result is just the spin operator, (35), apart from a factor $c$, so that

$$
\int d_{3} \mathbf{r} \hat{\mathbf{v}}_{\mathrm{rad}}=c \hat{\mathbf{S}}_{\mathrm{rad}}
$$

\section{Mode operators for conserved quantities}

Expressions (32)-(36) have a remarkably similar structure. They arise from products of the quantum fields given in Eqs. (27)-(30). After integration over position the terms containing products of two annihilation or two creation operators $\hat{a}_{\lambda^{\prime}} \hat{a}_{\lambda}$ or $\hat{a}_{\lambda^{\prime}}^{\dagger} \hat{a}_{\lambda}^{\dagger}$ vanish exactly. This is only the case since the expressions for the densities are invariant under the transformation, (25) and (26). As a result, each quantum operator $\hat{Q}_{\text {rad }}$ has the structure

$$
\hat{Q}_{\mathrm{rad}}=\frac{1}{2} \sum_{\lambda \lambda^{\prime}}^{\prime}\left[\hat{a}_{\lambda^{\prime}} \hat{a}_{\lambda}^{\dagger}+\hat{a}_{\lambda}^{\dagger} \hat{a}_{\lambda^{\prime}}\right]\left(\hat{Q}_{\mathrm{mod}}\right)_{\lambda \lambda^{\prime}}
$$

The last factor $\left(\hat{Q}_{\text {mod }}\right)_{\lambda \lambda^{\prime}}=\left\langle\mathbf{G}_{\lambda}\left|\hat{Q}_{\text {mod }}\right| \mathbf{G}_{\lambda^{\prime}}\right\rangle$ is the matrix element between the modes $\mathbf{G}_{\lambda}$ and $\mathbf{G}_{\lambda^{\prime}}$ of an operator $\hat{Q}_{\text {mod }}$ acting on mode space. For each one of these operators, off-diagonal elements with $\lambda \neq \lambda^{\prime}$ only occur between modes with the same value of the wave number $k_{\lambda}=k_{\lambda^{\prime}}$.

We denote the mode operators corresponding to the quantum operators (32)-(35) as $\hat{U}_{\text {mod }}, \hat{H}_{\text {mod }}, \hat{\mathbf{P}}_{\text {mod }}, \hat{\mathbf{L}}_{\text {mod }}$, and $\hat{\mathbf{S}}_{\text {mod }}$. Comparison of Eqs. (32), (33), and (36) with Eq. (38) shows that the mode operators for the energy, momentum, and helicity are defined by their action on modes:

$$
\begin{aligned}
& \hat{U}_{\mathrm{mod}} \mathbf{G}_{\lambda}=\hbar c k_{\lambda} \mathbf{G}_{\lambda}, \quad \hat{\mathbf{P}}_{\mathrm{mod}} \mathbf{G}_{\lambda}=\frac{\hbar}{i} \nabla \mathbf{G}_{\lambda}, \\
& \hat{H}_{\mathrm{mod}} \mathbf{G}_{\lambda}=\frac{\hbar}{k_{\lambda}} \nabla \times \mathbf{G}_{\lambda} .
\end{aligned}
$$

The right-hand sides of these identities are transverse vector fields, so that the operators $\hat{U}_{\text {mod }}$ and $\hat{H}_{\text {mod }}$ and each component of the vector operator $\hat{\mathbf{P}}_{\text {mod }}$ act within mode space. These operators are the same as the operators that represent observables of a single quantum particle with spin 1 and act 
on the wave function in coordinate representation. In spite of the quantum-mechanical appearance of these operators, their significance is just as classical as the modes. The expression for the helicity mode operator $\hat{H}_{\text {mod }}$ was mentioned in a different context by Fernandez-Corbaton et al. [8]. Since $\nabla \times\left(\nabla \times \mathbf{G}_{\lambda}\right) / k_{\lambda}^{2}=\mathbf{G}_{\lambda}$, the square of the mode operator for helicity is the c-number $\hbar^{2}$, so that $\hat{H}_{\text {mode }}$ can only have eigenvalues $\pm \hbar$.

It is obvious from Eqs. (34) and (35) that the quantum vector operator $\hat{\mathbf{J}}_{\text {rad }}=\hat{\mathbf{L}}_{\text {rad }}+\hat{\mathbf{S}}_{\text {rad }}$ for the total optical angular momentum corresponds to the mode operator $\hat{\mathbf{J}}_{\text {mod }}=\hat{\mathbf{L}}_{\text {mod }}+$ $\hat{\mathbf{S}}_{\text {mod }}$. The operator $\hat{\mathbf{L}}_{\text {mod }}$ acts on the $\mathbf{r}$ dependence, while the operator $\hat{\mathbf{S}}_{\text {mod }}$ is a vector of three $3 \times 3$ matrices acting exclusively on the vector nature of a mode. They are defined by the identities [11]

$$
\hat{\mathbf{L}}_{\mathrm{mod}} \mathbf{G}_{\lambda}=\frac{\hbar}{i}(\mathbf{r} \times \nabla) \mathbf{G}_{\lambda}, \mathbf{G}_{\lambda}^{*} \cdot \hat{\mathbf{S}}_{\mathrm{mod}} \cdot \mathbf{G}_{\lambda^{\prime}}=\frac{\hbar}{i} \mathbf{G}_{\lambda}^{*} \times \mathbf{G}_{\lambda^{\prime}}
$$

These mode operators $\hat{\mathbf{L}}_{\text {mod }}$ and $\hat{\mathbf{S}}_{\text {mod }}$ have the same form as the quantum operators for the orbital angular momentum and spin of a particle with spin 1 [29]. They obey the commutation rules for angular momentum, in the general form

$$
\left[\hat{J}_{i}, \hat{J}_{j}\right]=i \hbar \sum_{k} \epsilon_{i j k} \hat{J}_{k}
$$

with $\epsilon_{i j k}$ the fully antisymmetric tensor. Each component of the mode operator $\hat{\mathbf{J}}_{\text {mod }}$ maps a transverse mode into another transverse vector field. However, as noted a while ago $[11,13,23,30]$, this is not true for the separate mode operators $\hat{\mathbf{L}}_{\text {mod }}$ and $\hat{\mathbf{S}}_{\text {mod }}$, since they do not preserve the transversality of the modes. Nevertheless, the corresponding quantum operators, (34) and (35), remain fully decent vector operators on the Fock space of states of the quantized radiation field. These quantum operators only contain matrix elements of the mode operators, (41), between transverse vector fields.

\section{TRANSFORMATION OPERATORS}

The quantum operators, (32)-(36), are generators of symmetry transformations of the radiation field operators, (27)(30). For example, the result of a symmetry transformation generated by $\hat{Q}_{\text {rad }}$ applied to the vector potential $\hat{\mathbf{A}}$ is determined by the replacement

$$
\hat{\mathbf{A}}^{+} \rightarrow \exp \left(i \alpha \hat{Q}_{\mathrm{rad}}\right) \hat{\mathbf{A}}^{+} \exp \left(-i \alpha \hat{Q}_{\mathrm{rad}}\right),
$$

with $\alpha$ the relevant transformation parameter. This transformation is determined by the commutator $\left[\hat{Q}_{\mathrm{rad}}, \hat{\mathbf{A}}^{+}\right]$. Recall that the action of the quantum operators $\hat{Q}_{\text {rad }}$ is governed by the commutation rule of the annihilation and creation operators. Since $\hat{\mathbf{A}}^{+}$is a linear combination of sums of the type $\sum_{\lambda} \hat{a}_{\lambda} \mathbf{G}_{\lambda}$, we need the commutator

$$
\begin{aligned}
\sum_{\lambda} \mathbf{G}_{\lambda}\left[\hat{Q}_{\mathrm{rad}}, \hat{a}_{\lambda}\right] & =-\sum_{\lambda \lambda^{\prime}} \mathbf{G}_{\lambda^{\prime}}\left(Q_{\mathrm{mod}}\right){ }_{\lambda^{\prime} \lambda} \hat{a}_{\lambda} \\
& =-\sum_{\lambda} \hat{a}_{\lambda}\left(\hat{Q}_{\mathrm{mod}} \mathbf{G}_{\lambda}\right),
\end{aligned}
$$

which follows from relation (38). The last equality sign in Eq. (44) only holds when the vector function $\hat{Q}_{\bmod } \mathbf{G}_{\lambda}$ can be expanded in modes. However, this is true only when this operator $\hat{Q}_{\text {mod }}$ preserves the transversality of vector fields. For the operators $\hat{\mathbf{L}}_{\text {mod }}$ and $\hat{\mathbf{S}}_{\text {mod }}$ the transverse modes $\mathbf{G}_{\lambda}$ do not represent a closed set, and this step is not justified. For the quantities energy, momentum, and helicity Eq. (44) is correct, and we may conclude that

$$
\exp \left(i \alpha \hat{Q}_{\mathrm{rad}}\right) \hat{\mathbf{A}}^{+} \exp \left(-i \alpha \hat{Q}_{\mathrm{rad}}\right)=\exp \left(-i \alpha \hat{Q}_{\mathrm{mod}}\right) \hat{\mathbf{A}}^{+} \text {. }
$$

This can be checked by taking the derivative of (45) with respect to $\alpha$. The other fields, $\hat{\mathbf{B}}^{+}, \hat{\mathbf{E}}_{\perp}^{+}$, and $\hat{\mathbf{C}}^{+}$, transform in the same way. We conclude that the symmetry transformation generated by these quantities has two possible representations. It can be expressed either in terms of the quantum operators $\hat{Q}_{\text {rad }}$ or by transforming the mode functions $\mathbf{G}_{\lambda}$ in terms of the mode operators $\hat{Q}_{\text {mod }}$. For the operators $\hat{\mathbf{L}}_{\text {mod }}$ and $\hat{\mathbf{S}}_{\text {mod }}$ the transverse modes $\mathbf{G}_{\lambda}$ do not represent a closed set, and Eq. (45) does not hold.

\section{A. Time evolution, translation, and rotation}

The free time evolution of the fields is generated by the energy operator, and it is described by Eq. (43), with the substitution $\alpha \hat{Q}_{\text {rad }} \rightarrow \hat{U}_{\text {rad }} t / \hbar$. This represents the time evolution of a physical quantity in the Heisenberg representation. For all field operators the effect of the evolution is that the operators $\hat{a}_{\lambda}$ and $\hat{a}_{\lambda}^{\dagger}$ are multiplied by the oscillating factors $\exp \left(\mp i \omega_{\lambda} t\right)$. The resulting expressions for the time-dependent fields can also be obtained by applying the mode operator $\hat{U}_{\text {mod }}$, in accordance with Eq. (45). Then the oscillating factors arise from the mode operator acting on the mode functions $\mathbf{G}_{\lambda}$. When we view the field as a wave function, this is reminiscent of the Schrödinger picture. In the presence of charges and currents, their interaction with the fields also contributes to the energy and, thereby, to the time dependence of the fields.

The momentum operator $\hat{\mathbf{P}}_{\text {rad }}$ generates translations of the field. This is described by Eq. (43), with the substitution $\alpha \hat{Q}_{\mathrm{rad}} \rightarrow \mathbf{b} \cdot \hat{\mathbf{P}}_{\mathrm{rad}} / \hbar$, where $\mathbf{b}$ is the displacement vector. The translation operators acting on quantum states and on mode functions are

$$
\begin{aligned}
\hat{T}_{\text {rad }}(\mathbf{b}) & =\exp \left(-i \mathbf{b} \cdot \hat{\mathbf{P}}_{\mathrm{rad}} / \hbar\right), \\
\hat{T}_{\text {mod }}(\mathbf{b}) & =\exp \left(-i \mathbf{b} \cdot \hat{\mathbf{P}}_{\mathrm{mod}} / \hbar\right)
\end{aligned}
$$

For the transformation of the absorptive part of the vector potential $\hat{\mathbf{A}}$ we find from Eq. (45) in this case

$$
\hat{T}_{\text {rad }}^{\dagger}(\mathbf{b}) \hat{\mathbf{A}}^{+}(\mathbf{r}) \hat{T}_{\text {rad }}(\mathbf{b})=\hat{T}_{\bmod }(\mathbf{b}) \hat{\mathbf{A}}^{+}(\mathbf{r}) \text {. }
$$

Again, the left-hand side has the form of a transformation of a quantum operator, while the right-hand side looks like the translation of a wave function in the Schrödinger picture. The latter picture shows that the transformed operator is equal to $\hat{\mathbf{A}}^{+}(\mathbf{r}-\mathbf{b})$, just as expected. The translation of the other fields, (28)-(30), takes a similar form. We see that the correct translation expression results either from the commutation rules of the bosonic operators or from the mode operator for momentum, acting on the mode functions.

Rotations of the radiation field are generated by the operator $\hat{\mathbf{J}}_{\text {rad }}$ for the angular momentum. We substitute $\alpha \hat{Q}_{\text {rad }} \rightarrow \boldsymbol{\beta}$. $\hat{\mathbf{J}}_{\text {rad }} / \hbar$ in Eq. (43), which results in a rotation with the rotation axis in the direction of the vector $\boldsymbol{\beta}$, over an angle $\beta=|\beta|$. In 
the mode representation, the components of the operator $\hat{\mathbf{S}}_{\text {mod }}$ commute with the components of $\hat{\mathbf{L}}_{\text {mod }}$, so that the rotation operator $\exp \left(-i \boldsymbol{\beta} \cdot \hat{\mathbf{J}}_{\text {mod }} / \hbar\right)$ is the product of a spin and an orbital rotation operator. When acting on the field operator $\hat{\mathbf{A}}^{+}$, the spin part describes a rotation of the vector. The effect of the orbital part is well known from standard quantum mechanics: the rotated wave function at the rotated position $\mathbf{r}$ has the same value as the original wave function at the original position $\mathbf{r}^{\prime}=\exp \left(i \boldsymbol{\beta} \cdot \hat{\mathbf{S}}_{\text {mod }} / \hbar\right) \mathbf{r}$. This gives

$$
\exp \left(-i \boldsymbol{\beta} \cdot \hat{\mathbf{J}}_{\text {mod }} / \hbar\right) \hat{\mathbf{A}}^{+}(\mathbf{r})=\exp \left(-i \boldsymbol{\beta} \cdot \hat{\mathbf{S}}_{\text {mod }} / \hbar\right) \hat{\mathbf{A}}^{+}\left(\mathbf{r}^{\prime}\right) \text {. }
$$

This rotated field operator, (48), is indeed divergence-free, just like the original operator $\hat{\mathbf{A}}^{+}$. Again, the rotation of the other field operators has the same form.

\section{B. Duality transformation}

Now we consider the symmetry transformation generated by the optical helicity $\hat{H}_{\text {rad }}$. Since the corresponding mode operator preserves transversality, we can apply relation (45), where we substitute $\alpha \hat{Q}_{\text {rad }} \rightarrow \phi \hat{H}_{\text {rad }} / \hbar$. For the absorptive parts of the fields, the commutation rule, (44), then takes the form

$$
\frac{i}{\hbar}\left[\hat{H}_{\mathrm{rad}}, \hat{\mathbf{A}}^{+}\right]=-\frac{i}{\hbar} \hat{H}_{\text {mod }} \hat{\mathbf{A}}^{+} .
$$

When we apply this identity to the electric and the magnetic field, while using the operator expression in Eq. (40), we find

$$
\frac{1}{\hbar} \hat{H}_{\text {mod }} \hat{\mathbf{E}}_{\perp}^{+}=i c \hat{\mathbf{B}}^{+}, \quad \frac{1}{\hbar} \hat{H}_{\mathrm{mod}} c \hat{\mathbf{B}}^{+}=-i \hat{\mathbf{E}}_{\perp}^{+} .
$$

For the vector potentials in Eqs. (27) and (30) we obtain

$$
\frac{1}{\hbar} \hat{H}_{\bmod } c \hat{\mathbf{A}}^{+}=i \hat{\mathbf{C}}^{+}, \quad \frac{1}{\hbar} \hat{H}_{\bmod } \hat{\mathbf{C}}^{+}=-i c \hat{\mathbf{A}}^{+} .
$$

These equations confirm that the square of the helicity mode operator is the unit operator times $\hbar^{2}$, so that its eigenvalues are $\pm \hbar$. We denote the transformation operators

$$
\begin{aligned}
\hat{D}_{\text {rad }}(\phi) & =\exp \left(-i \phi \hat{H}_{\mathrm{rad}} / \hbar\right), \\
\hat{D}_{\text {mod }}(\phi) & =\exp \left(-i \phi \hat{H}_{\mathrm{mod}} / \hbar\right),
\end{aligned}
$$

where the parameter $\phi$ has the dimension of an angle. Then we find the integral form of Eq. (50):

$$
\begin{aligned}
\hat{D}_{\mathrm{rad}}^{\dagger}(\phi) \hat{\mathbf{E}}_{\perp}^{+} \hat{D}_{\mathrm{rad}}(\phi) & =\hat{D}_{\mathrm{mod}}(\phi) \hat{\mathbf{E}}_{\perp}^{+} \\
& =\hat{\mathbf{E}}_{\perp}^{+} \cos \phi+c \hat{\mathbf{B}}^{+} \sin \phi \\
\hat{D}_{\mathrm{rad}}^{\dagger}(\phi) c \hat{\mathbf{B}}^{+} \hat{D}_{\mathrm{rad}}(\phi) & =\hat{D}_{\mathrm{mod}}(\phi) c \hat{\mathbf{B}}^{+} \\
& =-\hat{\mathbf{E}}_{\perp}^{+} \sin \phi+c \hat{\mathbf{B}}^{+} \cos \phi .
\end{aligned}
$$

This is just the quantum version of the statement that the helicity operator generates the dual symmetry of Maxwell's equations in free space: a solution is transformed into another solution when $\mathbf{E}$ and $c \mathbf{B}$ are replaced by the linear combinations given in (25) [7,9,13,31,32]. A similar transformation holds for the vector potentials. In the quantum picture, this symmetry is based on the fact that the helicity operator $\hat{H}_{\text {rad }}$ commutes with the Hamiltonian $\hat{U}_{\text {rad }}$ of the free field. The quantum expressions for the densities of the energy $\hat{u}_{\text {rad }}$, momentum $\hat{\mathbf{p}}_{\text {rad }}$, angular momentum $\hat{\mathbf{j}}_{\text {rad }}$, chirality $\hat{\chi}_{\text {rad }}$, and helicity $\hat{h}_{\text {rad }}$ are all invariant under the dual transformation.

\section{Spin rotation and commutation rules}

We have seen that the general relations (44) and (45) do not hold for the operators $\hat{\mathbf{L}}_{\text {mod }}$ and $\hat{\mathbf{S}}_{\text {mod. }}$ The reason is that these operators do not preserve the transversality of modes. We want to argue that we can salvage these relations when we replace the spin operator $\hat{\mathbf{S}}_{\text {mod }}$ with the vector operator $\hat{\boldsymbol{\Sigma}}$, defined by

$$
\hat{\mathbf{\Sigma}} \mathbf{G}_{\lambda}=\frac{\hbar}{i} \frac{1}{k_{\lambda}^{2}} \nabla\left(\nabla \times \mathbf{G}_{\lambda}\right)=\frac{1}{i k_{\lambda}} \nabla\left(\hat{H}_{\mathrm{mod}} \mathbf{G}_{\lambda}\right) .
$$

This Hermitian mode operator preserves transversality, and its form nicely agrees with the picture of the helicity as the spin component in the direction of propagation (or momentum). Moreover, expression (54) can be conjectured by relation (37) between the helicity flux and the spin of the radiation field.

Mathematically, the operator $\hat{\boldsymbol{\Sigma}}$ may be viewed as the projection of the spin operator $\hat{\mathbf{S}}_{\text {mod }}$ on transverse vector functions. In order to prove this, we take as the basis of modes the circularly polarized plane-wave modes

$$
\mathbf{G}_{\mathbf{k} \pm}(\mathbf{r})=\frac{\mathbf{e}_{\mathbf{k} \pm}}{\sqrt{V}} \exp (i \mathbf{k} \cdot \mathbf{r}),
$$

with $V=L^{3}$ a cubic quantization volume. The components of the wave vectors $\mathbf{k}$ are integer multiples of $2 \pi / L$. Here the mode index attains the values $\lambda \equiv\{\mathbf{k}, \pm\}$, where $\mathbf{e}_{\mathbf{k} \pm}$ indicates the two opposite circular polarization vectors normal to $\mathbf{k}$. We denote as $\boldsymbol{\kappa} \equiv \mathbf{k} / k$ the unit vector in the direction of $\mathbf{k}$. By direct substitution one shows that the plane-wave modes $\mathbf{G}_{\mathbf{k} \pm}$ are eigenmodes of the components $\hat{\Sigma}_{j}$ of the operator, (54), with eigenvalue $\pm \hbar \kappa_{j}$.

In Eq. (35) only the terms with $\mathbf{k}=\mathbf{k}^{\prime}$ survive the integration, so that $\hat{\mathbf{S}}_{\text {mod }}$ is diagonal in $\mathbf{k}$. The circular polarization vectors obey the relations $\mathbf{e}_{\mathbf{k}-}^{*} \times \mathbf{e}_{\mathbf{k}+}=0$ and $\mathbf{e}_{\mathbf{k} \pm}^{*} \times \mathbf{e}_{\mathbf{k} \pm}=$ $\pm i \kappa$. This means that the only nonvanishing matrix elements of the mode operator $\hat{\mathbf{S}}_{\text {mod }}$ are the diagonal elements

$$
\left\langle\mathbf{G}_{\mathbf{k}, \pm}\left|\hat{\mathbf{S}}_{\text {mod }}\right| \mathbf{G}_{\mathbf{k}, \pm}\right\rangle= \pm \hbar \boldsymbol{\kappa} .
$$

Therefore, projected on the transverse modes, the operators $\hat{\mathbf{S}}_{\text {mod }}$ and $\hat{\boldsymbol{\Sigma}}$ coincide. This is remarkable, since the components of $\hat{\boldsymbol{\Sigma}}$ commute, whereas the components of $\hat{\mathbf{S}}_{\text {mod }}$ obey the angular-momentum commutation rules.

It is now obvious that Eqs. (43) and (44) still hold when we substitute $\hat{\mathbf{S}}_{\text {rad }}$ for $\hat{Q}_{\text {rad }}, \backslash$ and $\hat{\boldsymbol{\Sigma}}$ for $\hat{Q}_{\text {mod }}$. For spin rotation, the equivalence of Eq. (45) therefore reads

$$
\exp \left(i \boldsymbol{\beta} \cdot \hat{\mathbf{S}}_{\mathrm{rad}} / \hbar\right) \hat{\mathbf{A}}^{+} \exp \left(-i \boldsymbol{\beta} \cdot \hat{\mathbf{S}}_{\mathrm{rad}} / \hbar\right)=\exp (-i \boldsymbol{\beta} \cdot \hat{\mathbf{\Sigma}} / \hbar) \hat{\mathbf{A}}^{+} \text {. }
$$

This shows that the spin operator $\hat{\mathbf{S}}_{\text {rad }}$ generates rotations of the polarization vector for each plane-wave component separately, with the $\mathbf{k}$ vector as the axis, over an angle $\boldsymbol{\beta} \cdot \boldsymbol{\kappa}$.

The plane-wave modes are common eigenmodes of the operators, (39), (40), and (54), so that all their commutators vanish. $\hat{U}_{\text {mod }}$ has the eigenvalues $\hbar c k=\hbar \omega$, while the eigenvalues of the mode operator $\hat{\mathbf{P}}_{\text {mod }}$ are $\hbar \mathbf{k}$. The mode operator $\hat{H}_{\text {mod }}$ for helicity has the eigenvalues $\pm \hbar$, and the eigenvalues of $\hat{\boldsymbol{\Sigma}}$ are $\hbar \boldsymbol{\kappa}$. Commuting mode operators give rise to commuting quantum operators. The corresponding quantum operators are the sum over eigenmodes of the product of the number operator and the eigenvalue of the corresponding 
mode operator, as given by

$$
\begin{aligned}
\hat{U}_{\mathrm{rad}} & =\sum_{\mathbf{k}} \sum_{ \pm} \hbar c k \hat{N}_{\mathbf{k} \pm}, \\
\hat{\mathbf{P}}_{\mathrm{rad}} & =\sum_{\mathbf{k}} \sum_{ \pm} \hbar \mathbf{k} \hat{N}_{\mathbf{k} \pm}, \\
\hat{H}_{\mathrm{rad}} & =\sum_{\mathbf{k}} \hbar\left[\hat{N}_{\mathbf{k}+}-\hat{N}_{\mathbf{k}-}\right], \\
\hat{\mathbf{S}}_{\mathrm{rad}} & =\sum_{\mathbf{k}} \hbar \boldsymbol{\kappa}\left[\hat{N}_{\mathbf{k}+}-\hat{N}_{\mathbf{k}-}\right] .
\end{aligned}
$$

The last two expressions in (58) correspond to the intuitive picture that a circularly polarized photon in a plane-wave mode contributes to the electromagnetic spin a value $\pm \hbar$ in the direction of the wave vector $\mathbf{k}$. The expression for $\hat{\mathbf{S}}_{\mathrm{rad}}$ was obtained over 30 years ago by Lenstra and Mandel using different techniques [33]. A remarkable feature, which was only noticed much later [11], is that the three components of $\hat{\mathbf{S}}_{\mathrm{rad}}$ commute with each other, so that they do not obey the commutation relations for angular momentum. This means that the quantum operator $\hat{\mathbf{S}}_{\text {rad }}$ does not represent an angular momentum. So the components of the quantum vector operator $\hat{\mathbf{J}}_{\text {rad }}$ obey the commutation rules for angular momenta, whereas the components of $\hat{\mathbf{L}}_{\text {rad }}$ and $\hat{\mathbf{S}}_{\text {rad }}$ do not [30].

We have seen that the transversality-conserving mode operator $\hat{\boldsymbol{\Sigma}}$ for spin generates the quantum operator $\hat{\mathbf{S}}_{\text {rad }}$ for spin, in the sense of Eq. (38). A similarly modified mode operator exists that generates the quantum operator $\hat{\mathbf{L}}_{\text {rad }}$. This is the mode operator complementary to $\hat{\boldsymbol{\Sigma}}$, defined by

$$
\hat{\boldsymbol{\Lambda}}=\hat{\mathbf{J}}_{\bmod }-\hat{\boldsymbol{\Sigma}} \text {. }
$$

Since both $\hat{\mathbf{J}}_{\text {mod }}$ and $\hat{\boldsymbol{\Sigma}}$ preserve transversality, so does $\hat{\boldsymbol{\Lambda}}$, which is therefore the projection of the mode operator $\hat{\mathbf{L}}_{\text {mod }}$ on the space of transverse modes. Since the components of $\hat{\boldsymbol{\Sigma}}$ commute, while the components of $\hat{\mathbf{J}}_{\text {mod }}$ obey the standard commutation rule, (42), for angular momenta, the commutators involving $\hat{\boldsymbol{\Lambda}}$ have the modified form

$$
\begin{aligned}
& {\left[\hat{\Lambda}_{i}, \hat{\Lambda}_{j}\right]=i \hbar \sum_{k} \epsilon_{i j k}\left(\hat{\Lambda}_{k}-\hat{\Sigma}_{k}\right),} \\
& {\left[\hat{\Lambda}_{i}, \hat{\Sigma}_{j}\right]=i \hbar \sum_{k} \epsilon_{i j k} \hat{\Sigma}_{k} .}
\end{aligned}
$$

These commutation rules for the mode operators $\hat{\boldsymbol{\Lambda}}$ and $\hat{\boldsymbol{\Sigma}}$ are the same as for the quantum operators $\hat{\mathbf{L}}_{\text {rad }}$ and $\hat{\mathbf{S}}_{\text {rad }}[30]$.

\section{CONCLUSIONS}

In this paper we compare and derive continuity equations for conserved quantities of a classical electromagnetic field in the presence of sources. The pseudoscalar densities of the optical chirality and helicity are shown to obey continuity equations with a structure similar to that of the equation for the field energy. Whereas the exchange of energy between field and matter arises obviously from the work performed on the charges, the situation for helicity and chirality is different. The main reason is that there is no obvious analogous quantity for the sources. The loss rate of the chirality density is determined by the scalar product of the current density and the curl of the electric field. While the chirality density is locally determined by the fields, the helicity density is defined in terms of the magnetic and electric vector potentials, which depend on the fields at other positions. On the other hand, the helicity seems to be a quantity of a more fundamental nature, since it is also the generator of the dual symmetry transformation. Moreover, the integrated helicity flux is proportional to the total optical spin. Also, the helicity flux density obeys a continuity equation, which has a structure similar to that of the continuity equation for the optical momentum (which is proportional to the energy flux density). In the special case of monochromatic fields, the chirality and the helicity differ only by a constant factor.

The role of conserved quantities as generators of symmetry transformations is most straightforward in a quantum description. Quantum operators for the density of conserved quantities are obtained by substituting the quantum operators for the fields in the classical expressions. Since the conserved quantities are all quadratic in the fields, they take the form of a double summation over modes, as represented in Eq. (38). The summands contain matrix elements of classical operators acting on the modes. These mode operators resemble operators acting on the wave function of a single quantum particle with spin 1, although the fields they operate on are classical. They generate transformations of modes and, thereby, of classical fields. In this sense the step from the mode operators to the quantum operators is reminiscent of second quantization, leading to quantum field theories. In the case of electromagnetism, however, there is no preceding first quantization. For the field energy and momentum the picture is nonproblematic. The symmetry transformation describes the time evolution and spatial translation of the fields. The quantum helicity operator $\hat{H}_{\text {rad }}$ generates the duality transformation, (25), of the quantum fields. The corresponding mode operator $\hat{H}_{\text {mod }}$ is specified in (40). The operators for the angular momentum are more subtle. The orbital and spin parts of the mode operator do not preserve the transversality of the modes, while their sum does. We introduce an alternative mode operator for spin that coincides with the transverse projection of the vector of matrices for spin 1. The components of this vector operator have a common basis of eigenmodes, so that they commute. This also leads to an alternative mode operator for the orbital part of the angular momentum, with modified commutation rules. The expressions for both the quantum operators and the mode operators are independent of the basis of the modes.
[1] Y. Tang and A. E. Cohen, Phys. Rev. Lett. 104, 163901 (2010).

[2] K. Y. Bliokh and F. Nori, Phys. Rev. A 83, 021803 (2011).
[3] M. M. Coles and D. L. Andrews, Phys. Rev. A 85, 063810 (2012) 
[4] C. Rosales-Guzmán, K. Volke-Sepulveda, and J. P. Torres, Opt. Lett. 37, 3486 (2012).

[5] D. L. Andrews and M. M. Coles, Opt. Lett. 37, 3009 (2012).

[6] D. M. Lipkin, J. Math. Phys. 5, 696 (1964).

[7] I. Fernandez-Corbaton, X. Zambrana-Puyalto, and G. MolinaTerriza, Phys. Rev. A 86, 042103 (2012).

[8] I. Fernandez-Corbaton, X. Zambrana-Puyalto, N. Tischler, X. Vidal, M. L. Juan, and G. Molina-Terriza, Phys. Rev. Lett. 111, 060401 (2013).

[9] R. P. Cameron, S. M. Barnett, and A. M. Yao, New J. Phys. 14, 053050 (2012).

[10] K. Y. Bliokh, A. Y. Bekshaev, and F. Nori, New J. Phys. 15, 033026 (2013).

[11] S. J. van Enk and G. Nienhuis, J. Mod. Opt. 41, 963 (1994).

[12] K. Y. Bliokh, M. A. Alonso, E. A. Ostrovskaya, and A. Aiello, Phys. Rev. A 82, 063825 (2010).

[13] S. M. Barnett, J. Mod. Opt. 57, 1339 (2010).

[14] I. Białynicki-Birula and Z. Białynicka-Birula, J. Opt. 13, 064014 (2011).

[15] D. B. Ruffner and D. G. Grier, Phys. Rev. Lett. 108, 173602 (2012).

[16] K. Y. Bliokh, J. Dressel, and F. Nori, New J. Phys. 16, 093037 (2014).

[17] I. Białynicki-Birula, Progress in Optics, edited by E. Wolf (Elsevier, Amsterdam, 1996), Vol. 36.
[18] D. J. Griffiths, Introduction to Electrodynamics (Pearson, Boston, 2013).

[19] Y. Tang and A. E. Cohen, Science 332, 333 (2011).

[20] A. Canaguier-Durand and C. Genet, Phys. Rev. A 92, 043823 (2015).

[21] R. P. Cameron, S. M. Barnett, and A. M. Yao, New J. Phys. 16, 013020 (2014).

[22] H. K. Moffatt, J. Fluid Mech. 35, 117 (1969).

[23] C. Cohen-Tannoudji, J. Dupont-Roc, and G. Grynberg, Photons et Atomes (InterEditions CNRS, Paris, 1987) [in French]; Photons and Atoms (Wiley, New York, 1992).

[24] J. L. Trueba and A. F. Rañada, Eur. J. Phys. 17, 141 (1996).

[25] G. N. Afanasiev and Yu. Stepanovsky, Nuovo Cimento A 109, 271 (1996).

[26] L. Woltjer, Proc. Natl. Acad. Sci. USA 44, 489 (1958).

[27] H. K. Moffatt, J. Plasma Phys. 81, 905810608 (2015).

[28] S. M. Barnett, R. P. Cameron, and A. M. Yao, Phys. Rev A 86, 013845 (2012).

[29] I. Białynicki-Birula and Z. Białynicka-Birula, Opt. Commun. 264, 342 (2006).

[30] S. J. van Enk and G. Nienhuis, Europhys. Lett. 25, 497 (1994).

[31] M. G. Calkin, Am. J. Phys. 33, 958 (1965).

[32] R. P. Cameron and S. M. Barnett, New J. Phys. 14, 123019 (2012).

[33] D. Lenstra and L. Mandel, Phys. Rev. A 26, 3428 (1982). 